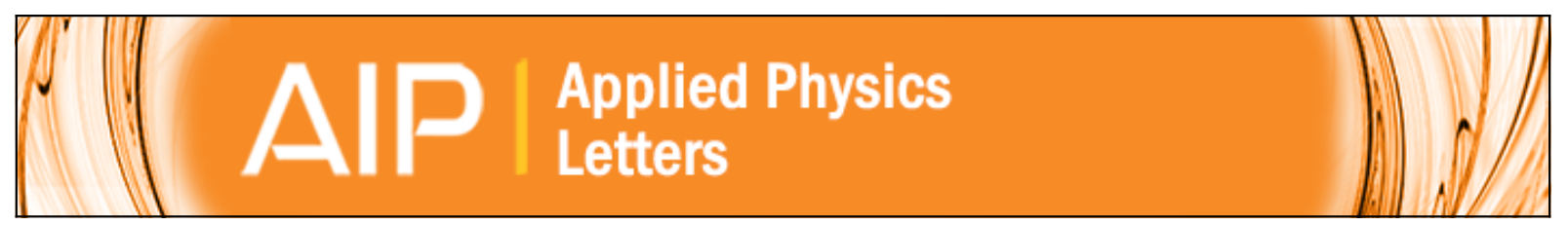

\title{
All-optical evaluation of spin-orbit interaction based on diffusive spin motion in a two- dimensional electron gas
}

M. Kohda, P. Altmann, D. Schuh, S. D. Ganichev, W. Wegscheider, and G. Salis

Citation: Applied Physics Letters 107, 172402 (2015); doi: 10.1063/1.4934671

View online: http://dx.doi.org/10.1063/1.4934671

View Table of Contents: http://scitation.aip.org/content/aip/journal/apl/107/17?ver=pdfcov

Published by the AIP Publishing

\section{Articles you may be interested in}

Energy-loss rate of a fast particle in two-dimensional semiconductors with Rashba spin-orbit coupling

Appl. Phys. Lett. 102, 052113 (2013); 10.1063/1.4790847

Realization of In $0.75 \mathrm{Ga} 0.25$ As two-dimensional electron gas bilayer system for spintronics devices based on Rashba spin-orbit interaction

J. Appl. Phys. 112, 113711 (2012); 10.1063/1.4766749

Intense terahertz laser fields on a two-dimensional electron gas with Rashba spin-orbit coupling

Appl. Phys. Lett. 86, 032107 (2005); 10.1063/1.1852732

Absorption of surface acoustic waves by a two-dimensional electron gas in the presence of spin-orbit interaction J. Appl. Phys. 94, 3229 (2003); 10.1063/1.1599631

Plasmons of a two-dimensional electron gas in the presence of spin orbit interaction Appl. Phys. Lett. 82, 724 (2003); 10.1063/1.1541098

\section{AIP $\left.\right|_{\text {APL Photonics }}$}

APL Photonics is pleased to announce Benjamin Eggleton as its Editor-in-Chief

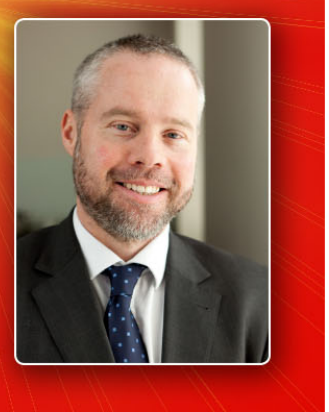




\title{
All-optical evaluation of spin-orbit interaction based on diffusive spin motion in a two-dimensional electron gas
}

\author{
M. Kohda,,${ }^{1,2}$ P. Altmann, ${ }^{1}$ D. Schuh, ${ }^{3}$ S. D. Ganichev, ${ }^{3}$ W. Wegscheider,${ }^{4}$ and G. Salis ${ }^{1}$ \\ ${ }^{1}$ IBM Research-Zürich, Säumerstrasse 4, CH-8803 Rüschlikon, Switzerland \\ ${ }^{2}$ Department of Materials Science, Tohoku University, 980-8579 Sendai, Japan \\ ${ }^{3}$ Institute of Experimental and Applied Physics, University of Regensburg, D-93040 Regensburg, Germany \\ ${ }^{4}$ Solid State Physics Laboratory, ETH Zürich, CH-8093 Zürich, Switzerland
}

(Received 17 September 2015; accepted 14 October 2015; published online 27 October 2015)

\begin{abstract}
A method is presented that enables the measurement of spin-orbit coefficients in a diffusive twodimensional electron gas without the need for processing the sample structure, applying electrical currents or resolving the spatial pattern of the spin mode. It is based on the dependence of the average electron velocity on the spatial distance between local excitation and detection of spin polarization, resulting in a variation of spin precession frequency that in an external magnetic field is linear in the spatial separation. By scanning the relative positions of the exciting and probing spots in a time-resolved Kerr rotation microscope, frequency gradients along the [100] and [010] crystal axes of GaAs/AlGaAs QWs are measured to obtain the Rashba and Dresselhaus spin-orbit coefficients, $\alpha$ and $\beta$. This simple method can be applied in a variety of materials with electron diffusion for evaluating spin-orbit coefficients. (C) 2015 AIP Publishing LLC.
\end{abstract}

[http://dx.doi.org/10.1063/1.4934671]

The spin-orbit (SO) interaction acts as an effective magnetic field for electron spins and enables the generation, manipulation, and detection of spin polarization, which are fundamental building blocks for future spintronic technologies. ${ }^{1,2}$ In III-V semiconductor heterostructures, two types of SO interactions exist: Rashba originating from structure inversion asymmetry in a quantum well $(\mathrm{QW})^{3}$ and Dresselhaus originating from bulk inversion asymmetry in the crystal structure. ${ }^{4}$ Under drift motion, electron spins move in a direction given by an applied electric field and therefore precess about a drift-induced SO effective magnetic field. ${ }^{5,6}$ By measuring this precession, the SO coefficients in a twodimensional electron gas (2DEG) can be determined by controlling the drift direction and speed. ${ }^{7,8}$ Even without application of a drift field, spins precess about SO fields on their diffusive paths. This leads to dephasing, ${ }^{9,10}$ but not to precession of the net spin polarization. After local spin excitation, a spatially varying spin rotation can be resolved as exemplified for the spin helix case ${ }^{11-13}$ and laterally confined wire structures. ${ }^{14,15}$ Such a spatial mapping of the spin mode allows the determination of the SO parameters, but requires a spatial resolution better than the SO length. Photogalvanic effects ${ }^{16}$ are sensitive to the ratio of the Rashba and the Dresselhaus field but require electric contacts to the 2DEG. Here, we introduce an optical method for characterizing SO fields that does not require electric contacts, works with arbitrary ratios of Rashba and Dresselhaus SO fields, and needs only modest spatial resolution where spot sizes may be larger than the SO length. It is based on the effect that the spin polarization probed at a different location from where it was excited moves on average with a velocity that points from the point of excitation to that of measurement. The probed spins therefore see an effective SO field that affects their precession frequency. We implemented this idea using a time-resolved Kerr rotation (TRKR) technique. The spin precession frequency of electrons under an external magnetic field is found to depend linearly on the spatial separation between the excitation and detection of spins. By analyzing this frequency gradient along two orthogonal axes of the 2DEG, both the Rashba and the Dresselhaus SO coefficients are evaluated. As we demonstrate, this method allows a precise determination of SO fields in unprocessed 2DEG samples without application of external electric fields.

The samples studied were 10-, 20-, and 30-nm-wide GaAs/AlGaAs QWs grown on (001) GaAs substrates. Each sample contains 15 equivalent QWs, and the carrier densities are $1.2,1.3$, and $1.7 \times 10^{15} \mathrm{~m}^{-2}$ for the $10-, 20-$, and $30-\mathrm{nm}$ QWs, respectively. ${ }^{17,18}$ The Dresselhaus SO coefficient, $\beta_{1}=-\gamma\left\langle k_{z}^{2}\right\rangle$, depends systematically on the QW width through its dependence on the expectation value of the squared wavenumber, $\left\langle k_{z}^{2}\right\rangle$, along the growth direction, $z$. The bulk value of the Dresselhaus SO coefficient is denoted by $\gamma$. The cubic Dresselhaus coefficient, $\beta_{3}=-\frac{1}{4} \gamma k_{\mathrm{F}}^{2}$, is subtracted from $\beta_{1}$ to obtain the total Dresselhaus coefficient $\beta=\beta_{1}-\beta_{3}$, where $k_{\mathrm{F}}$ is the Fermi wave number. The Rashba SO coefficient, $\alpha$, is controlled by the Si $\delta$-doping profile in the AlGaAs barrier layer and its absolute value is chosen to be smaller than $\beta$. In the TRKR measurement, a mode-locked Ti:sapphire laser generates 2-ps-long optical pulses with $79.2 \mathrm{MHz}$ repetition rate. The pulse train is split into pump and probe beams that are focused onto the sample surface [see Fig. 1(a)]. The circularly polarized pump pulses excite electron spins $\mathbf{S}$ polarized along the growth direction $z$ in a Gaussian distribution of sigma width $\sigma_{\mathrm{pp}}$ depicted in yellow. Linearly polarized probe pulses detect the out-of-plane spin component $S_{z}$ as a function of the delay time $t$ in a Gaussian spot shown in red in Fig. 1(a) (sigma width $\sigma_{\mathrm{pb}}$ ). The distance $r$ between the centers of the pump and probe spots can be controlled by a mirror. All measurements were taken at a temperature of $15 \mathrm{~K}$. Positive $x$ - and $y$-axes are defined along the [100] and [010] directions of the QW 
(a)

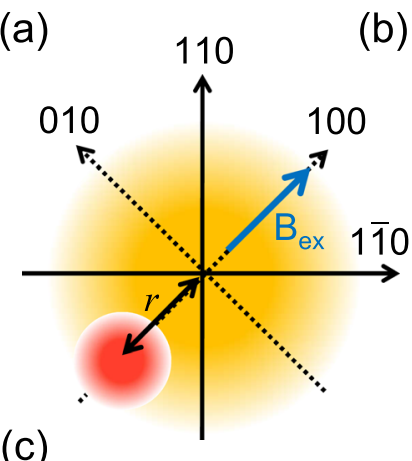

(b)

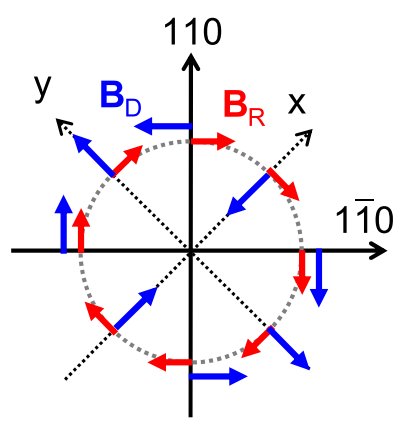

(c)

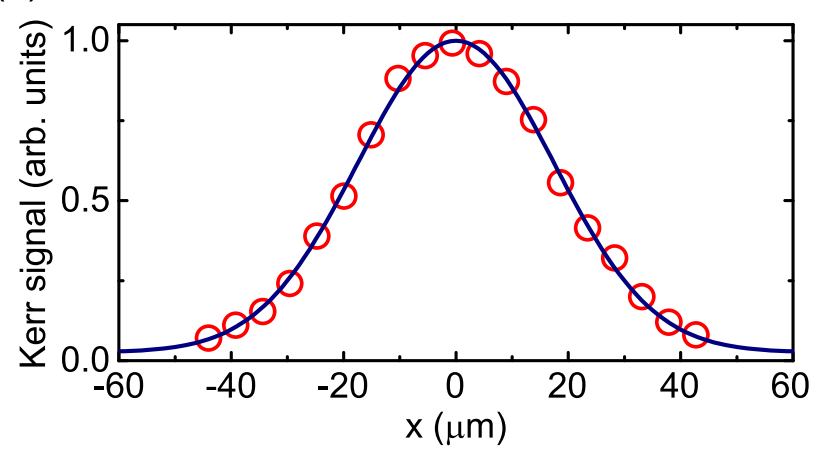

FIG. 1. (a) Schematic configuration of the positions of the pump (yellow) and the probe (red) spots for detecting the spatial modulation of the spin precession frequency. The scanning axes are the [100] and [010] directions under an inplane external magnetic field $\mathrm{B}_{\mathrm{ex}}$. The probe spot displacement $r$ along the [100] or [010] axis is defined as the distance between the centers of the probe and pump spots. (b) Directions of diffusion-induced SO fields for Rashba (red arrows) and Dresselhaus (blue arrows) SO interactions assuming $g<0, \beta>0$, and $\alpha<0$. (c) The spatial profile of normalized Kerr signal at $10 \mathrm{ps}$ after excitation with a pump sigma width $\sigma_{\mathrm{pp}}=18.0 \mu \mathrm{m}$ and probe sigma width $\sigma_{\mathrm{pb}}=3.5 \mu \mathrm{m}$ in a 20-nm-wide GaAs QW. Solid line is a fit by a Gaussian function.

samples. The pump spot size is changed from $\sigma_{\mathrm{pp}}=3$ to $30 \mu \mathrm{m}$, while the probe spot size is fixed to $\sigma_{\mathrm{pb}}=3-4 \mu \mathrm{m}$. Exemplarily, Fig. 1(c) shows the spatial profile of the normalized Kerr signal $10 \mathrm{ps}$ after excitation with $\sigma_{\mathrm{pp}}=18.0 \mu \mathrm{m}$ and $\sigma_{\mathrm{pb}}=3.5 \mu \mathrm{m}$ for displacement along the $x$-direction in a 20-nm GaAs/AlGaAs QW. The observed spin distribution is well fitted by a Gaussian function, see solid line in Fig. 1(c). The evaluated sigma width $\sigma_{\text {eff }}=18.3 \mu \mathrm{m}$ is a convolution of the pump and probe spots.

The mean velocity $v_{\mathrm{d}}$ of the electron spins excited within the pump spot that arrive at a time $t$ in the displaced probe spot is given by

$$
v_{\mathrm{d}}=\frac{2 D_{\mathrm{s}}}{2 D_{\mathrm{s}} t+\sigma_{\mathrm{eff}}^{2}} r
$$

where $D_{\mathrm{s}}$ is the spin-diffusion coefficient, and $\sigma_{\text {eff }}$ $=\sqrt{\sigma_{\mathrm{pp}}^{2}+\sigma_{\mathrm{pb}}^{2}}$ is the size of the convoluted spot size. Those electrons see an average SO field that depends on the direction and magnitude of $v_{\mathrm{d}}$. As a consequence of this field, a spatial displacement between the pump and probe spots leads to a spin precession. The strength of the SO field under such diffusive motion is described by the two contributions

$$
B_{\mathrm{R}}=\frac{2 \alpha}{g \mu_{\mathrm{B}}} \frac{m}{\hbar} v_{\mathrm{d}}
$$

$$
B_{\mathrm{D}}=\frac{2 \beta}{g \mu_{\mathrm{B}}} \frac{m}{\hbar} v_{\mathrm{d}},
$$

where $g$ is the electron $g$ factor, $\mu_{\mathrm{B}}$ is the Bohr magneton, $m$ is the electron effective mass, and $\hbar$ is the reduced Planck's constant. The directions of $B_{\mathrm{R}}$ and $B_{\mathrm{D}}$ as a function of the direction of $v_{\mathrm{d}}$ are depicted in Fig. 1(b). By applying an external magnetic field $B_{\mathrm{ex}} \gg B_{\mathrm{R}}, B_{\mathrm{D}}$ along either the $x$ or the $y$ direction, we map out frequency changes related to the projection of the SO field along $\mathbf{B}_{\text {ex }}$. For small times and large spot sizes $\left(\sigma_{\text {eff }}^{2} \gg 2 D_{\mathrm{s}} t\right)$, the SO field is constant and induces spin precession with a constant frequency. Our method works in this limit of large spot sizes. In the opposite limit of small spot sizes and long times, $v_{\mathrm{d}}$ and thus the SO field are proportional to $1 / t$. Then, spins do not precess in time but exhibit a spatial oscillation characteristic of the evolving spin mode.

To validate our idea, we first perform a Monte Carlo (MC) numerical simulation to calculate the spin-diffusion dynamics, specifically the spin precession frequency $\Omega$ for diffused electron spins under Rashba and Dresselhaus fields, $\mathbf{B}_{\mathrm{R}}$ and $\mathbf{B}_{\mathrm{D}}$. Details of the simulation are described in Ref. [19]. We take the following parameter values: $\sigma_{\mathrm{pp}}=4 \mu \mathrm{m}$, $\sigma_{\mathrm{pb}}=3.3 \mu \mathrm{m}, B_{\mathrm{ex}}=+1.0 \mathrm{~T}$ along $x, \alpha=-0.21 \times 10^{-13} \mathrm{eVm}$, $\beta_{1}=1.58 \times 10^{-13} \mathrm{eVm}, \quad \beta_{3}=0.27 \times 10^{-13} \mathrm{eVm}, \quad$ electron scattering time $\tau=0.49 \mathrm{ps}$, electron $g$ factor $g=-0.35$, and carrier density $N_{s}=1.5 \times 10^{11} \mathrm{~cm}^{-2}$. These values lead to a configuration of $\mathbf{B}_{\mathrm{R}}, \mathbf{B}_{\mathrm{D}}$, and $\mathbf{B}_{\mathrm{ex}}$ as shown in Fig. 2(a). Corresponding $D_{\mathrm{s}}$ and Dyakonov Perel spin relaxation time become $0.0069 \mathrm{~m}^{2} / \mathrm{s}$ and $1.28 \mathrm{~ns}$, respectively. The simulated time evolution of $S_{z}$ due to the external and the SO fields is averaged within a probe Gaussian spot and fitted by $S_{z 0} \exp \left(-t / \tau_{s}\right) \cos (\Omega t)$, where $S_{z 0}$ is the initial spin component along $z$, and $\tau_{s}$ is the spin relaxation time. The $\Omega$ obtained depends on the spatial displacement of the probe spot, indicating the contributions of SO fields due to both $\mathbf{B}_{\mathrm{R}}$ and $\mathbf{B}_{\mathrm{D}}$. Because $B_{\mathrm{ex}} \gg B_{\mathrm{R}}$ or $B_{\mathrm{D}}$, we obtain the components of $\mathbf{B}_{\mathrm{R}}+\mathbf{B}_{\mathrm{D}}$ parallel to $\mathbf{B}_{\mathrm{ex}}$ by considering $\Omega_{\mathrm{SOI}}=\Omega-\Omega_{\mathrm{ex}}$ with $\Omega_{\mathrm{ex}}=g \mu_{\mathrm{B}} B_{\mathrm{ex}} / \hbar$. We plot $\Omega_{\mathrm{SOI}}$ as a function of $x$ and $y$ in Fig. 2(b). Both crystal directions exhibit a linear change of $\Omega_{\mathrm{SOI}}$, reflected by the linear $v_{d}$ change with $x$
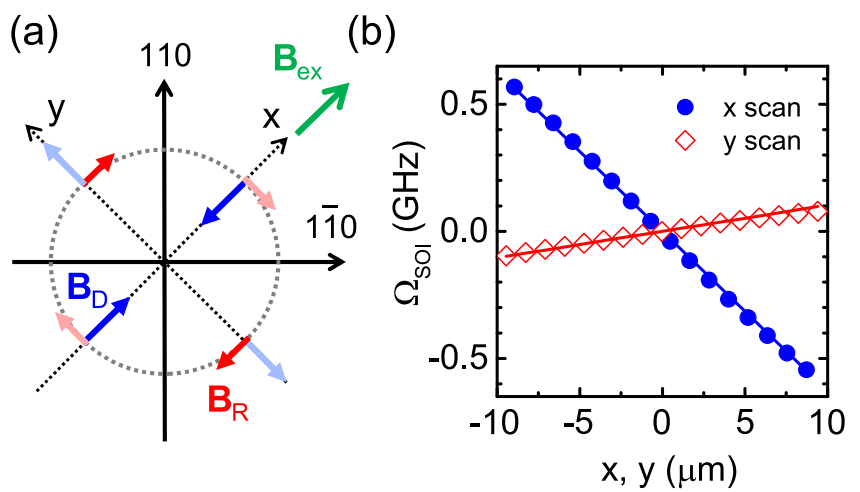

FIG. 2. (a) Configuration of the effective magnetic fields $B_{R}$ (red arrows) and $\mathrm{B}_{\mathrm{D}}$ (blue arrows) and external magnetic field $\mathrm{B}_{\mathrm{ex}}$ (green arrow) with respect to the crystal orientation for MC simulation. (b) Spatially modulated frequency components $\Omega_{\mathrm{SOI}}=\Omega-\Omega_{\mathrm{ex}}$ due to the SO fields, obtained from MC simulation with $B_{\mathrm{ex}}=+1.0 \mathrm{~T}$, plotted as a function of $x$ - and $y$-displacements of the probe spot. Solid lines are calculated from Eq. (3). 
and $y$ (see Eq. (1)). As seen in Fig. 2(a), $\mathbf{B}_{\mathrm{D}}\left(\mathbf{B}_{\mathrm{R}}\right)$ points along $\mathbf{B}_{\mathrm{ex}}$ for scans along the $x(y)$ direction, whereas the other field is perpendicular to $\mathbf{B}_{\text {ex }}$. Therefore, the spatial modulation of $\Omega_{\text {SOI }}$ is only sensitive to $\mathbf{B}_{\mathrm{D}}\left(\mathbf{B}_{\mathrm{R}}\right)$. The opposite $\Omega_{\text {SOI }}$ slope is due to the sign of $\mathbf{B}_{\mathrm{R}}$ and $\mathbf{B}_{\mathrm{D}}$ with respect to $\mathbf{B}_{\mathrm{ex}}$. As $\Omega_{\mathrm{SOI}}=g \mu_{\mathrm{B}} B_{\mathrm{R}(\mathrm{D})} / \hbar$, an analytical expression of $\Omega_{\mathrm{SOI}}$ is obtained by using Eqs. (1) and (2)

$$
\begin{aligned}
& \Omega_{\mathrm{DSOI}}(x)=-\frac{4 m D_{\mathrm{s}} \beta}{\hbar^{2}\left(2 D_{\mathrm{s}} \tau_{\mathrm{s}}+\sigma_{\mathrm{eff}}^{2}\right)} x \\
& \Omega_{\mathrm{RSOI}}(y)=-\frac{4 m D_{s} \alpha}{\hbar^{2}\left(2 D_{\mathrm{s}} \tau_{\mathrm{s}}+\sigma_{\mathrm{eff}}^{2}\right)} y .
\end{aligned}
$$

Owing to the diffusive expansion of the spin distribution profile with time $t, \Omega$ depends slightly on $t$. Because $\sigma_{\text {eff }}^{2} \gg 2 D_{\mathrm{s}} t$, this effect is small enough such that we can fit the data with a constant $\Omega$. The $\Omega$ obtained in this way is an average of the frequencies within the observed time window. Because of the exponential decay of the spin polarization, earlier times are weighted more. It is therefore a good approximation to replace $t$ in Eq. (1) by the spin lifetime $\tau_{s}$, which we did to obtain Eq. (3). The solid lines in Fig. 2(b) are calculated using Eq. (3) with the parameters used in the MC simulation. The analytical formulae reproduce the MC result remarkably well. This demonstrates that the SO fields can be extracted from the spatial modulation of the spin precession frequency.

We experimentally measured TRKR traces along the $x$ - and $y$-axes with $B_{\mathrm{ex}}=+1.0 \mathrm{~T}$ along $x$, which is the same configuration as in the simulation of Fig. 2(a). Figure 3(a) shows TRKR traces at $x=-29,0$, and $+28 \mu \mathrm{m}$ for a $20-\mathrm{nm}$ $\mathrm{QW}$, taken with $\sigma_{\mathrm{pp}}=18 \mu \mathrm{m}$. From positive to negative $x, \Omega$ increases. As there is no contribution of $v_{\mathrm{d}}$ at $x=0 \mu \mathrm{m}$, the $g$ factor is obtained directly from $\Omega,|g|=0.34$, which is consistent with the previous results. ${ }^{17,18}$ To analyze this spatial modulation of $\Omega$, the $\Omega_{\mathrm{SOI}}$ evaluated is plotted as a function of $x$ and $y$ in Fig. 3(b). As expected, we observe a linear change of $\Omega_{\text {SOI }}$ with displacement. The spatial modulation of the spin precession frequency, $\mathrm{d} \Omega_{\mathrm{SOI}} / \mathrm{d} r$, exhibits opposite slopes along the $x$ - and $y$-scans, indicating opposite signs of the Rashba and Dresselhaus SO coefficients.

We now verify that according to Eqs. (1) and (3), $v_{\mathrm{d}}$ and thus $\Omega_{\text {SOI }}$ depend on the convoluted Gaussian spot size, $\sigma_{\text {eff. }}$. We systematically measured $\mathrm{d} \Omega_{\mathrm{SOI}} / \mathrm{d} r$ for different pump spot sizes and plotted it as a function of $1 /\left(2 D_{\mathrm{s}} \tau_{\mathrm{s}}+\sigma_{\text {eff }}^{2}\right)$ in Fig. 3(c) (black symbols). A linear dependence with opposite slopes is observed for the $x$ - and $y$-directions and is fitted very well by Eq. (3) (dashed lines). The slopes correspond to $4 m D_{\mathrm{s}} \beta / \hbar^{2}$ and $4 m D_{\mathrm{s}} \alpha / \hbar^{2}$ for displacements along the $x$ - and $y$-axes, respectively. Values for $D_{\mathrm{s}}$ can be determined from the spatially resolved expansion of the spin polarization. Using $D_{\mathrm{s}}=0.0078 \mathrm{~m}^{2} / \mathrm{s},{ }^{18}$ we obtain $\beta=1.19 \times 10^{-13}$ and $\alpha=-1.6 \times 10^{-14} \mathrm{eVm}$. The signs of $\beta$ and $\alpha$ are determined from the direction of $\mathbf{B}_{\mathrm{R}}$ and $\mathbf{B}_{\mathrm{D}}$ with respect to $\mathbf{B}_{\mathrm{ex}}$. Red symbols in Fig. 3(c) correspond to MC-simulated values based on the parameters obtained, which reproduce the measurements remarkably well.

As d $\Omega_{\mathrm{SOI}} / \mathrm{d} r$ is proportional to the projection of $\mathbf{B}_{\mathrm{R}}+\mathbf{B}_{\mathrm{D}}$ onto $\mathbf{B}_{\mathrm{ex}}$, its value and sign should depend on the relative (a)

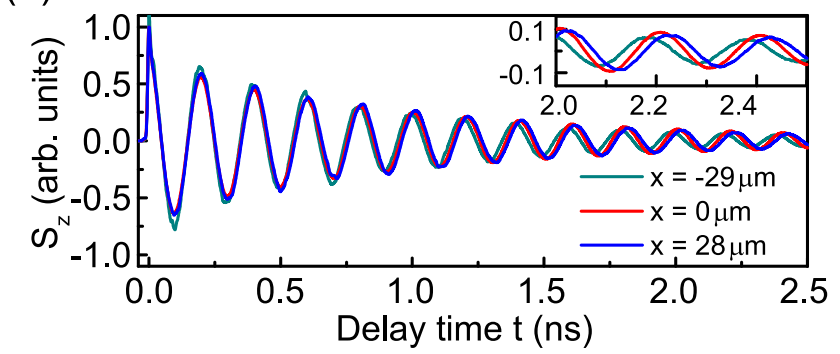

(b)

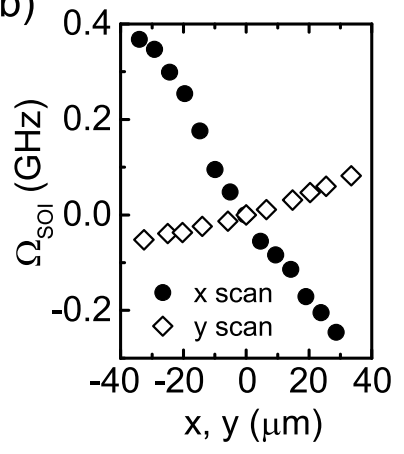

(c)

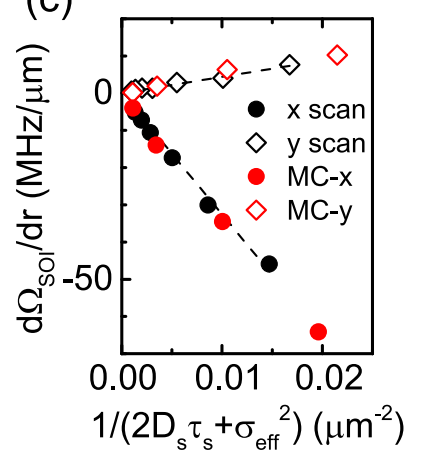

FIG. 3. (a) Measured spin polarization $S_{z}$ as a function of the delay time $t$ at $x=-29,0$, and $+28 \mu \mathrm{m}$ and with $B_{\mathrm{ex}}=+1.0 \mathrm{~T}$ applied along the $x$-direction in a 20-nm-wide GaAs QW. As seen in the magnified inset, the precession frequency increases from positive to negative $x$. (b) Spatially modulated frequency component $\Omega_{\mathrm{SOI}}$ as a function of the probe positions $x$ (filled circles) and $y$ (empty diamonds). (c) The measured (black symbols) and simulated (red symbols) spin precession frequency gradient, $\mathrm{d} \Omega_{\mathrm{SOI}} / \mathrm{d} r$, as a function of $1 /\left(2 D_{\mathrm{s}} \tau_{\mathrm{s}}+\sigma_{e f f}^{2}\right)$, where $r$ is either $x$ or $y$ depending on the scan axis. Dashed lines are fits based on Eq. (3).

directions between $\mathbf{B}_{\mathrm{R}}+\mathbf{B}_{\mathrm{D}}$ and $\mathbf{B}_{\mathrm{ex}}$. We apply $B_{\mathrm{ex}}=+1.0 \mathrm{~T}$ along different crystal directions, $\pm x$ and $+y$, and measure the dependence of $\mathrm{d} \Omega_{\mathrm{SOI}} / \mathrm{d} r$ on the pump spot size in a 20-nm QW [Fig. 4(a)]. Filled circles correspond to $\mathrm{d} \Omega_{\text {SOI }} / \mathrm{d} x$, empty diamonds to $\mathrm{d} \Omega_{\mathrm{SOI}} / \mathrm{d} y$. The relations of $\mathbf{B}_{\mathrm{R}}, \mathbf{B}_{\mathrm{D}}$, and $\mathbf{B}_{\mathrm{ex}}$ with respect to the scan axes are shown in Fig. 4(b). Scans along $x$ or $y$ measure either $B_{\mathrm{R}}$ or $B_{\mathrm{D}}$ depending on the exact configuration. For $\mathbf{B}_{\mathrm{ex}} \| \pm x$ (black and orange (a)

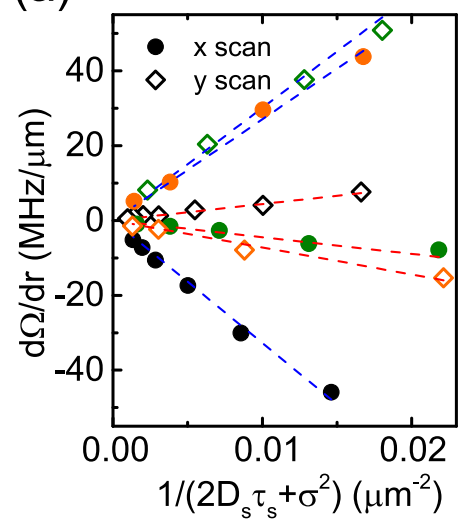

(b)

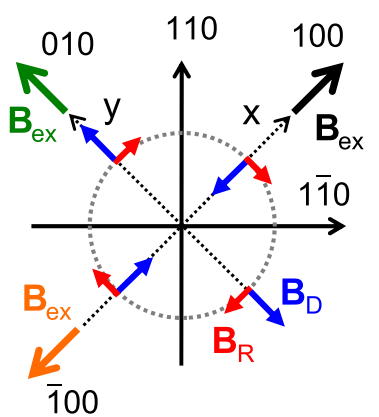

$\overline{1} 00$
FIG. 4. (a) The spin precession frequency gradient $\mathrm{d} \Omega_{\mathrm{SOI}} / \mathrm{d} r$ as a function of $1 /\left(2 D_{\mathrm{s}} \tau_{\mathrm{s}}+\sigma_{\text {eff }}^{2}\right)$ for $x$ (filled circles) and $y$ (empty diamonds) scans under different $\mathrm{B}_{\mathrm{ex}}$ directions; $B_{\mathrm{ex}}=1 \mathrm{~T}$ along $+x$ (black), along $+y$ (green), and along $-x$ (orange). Dashed blue and red lines are fits based on Eq. (3). (b) Relative directions of the effective magnetic fields, $\mathrm{B}_{\mathrm{R}}$ and $\mathrm{B}_{\mathrm{D}}$, and directions of the external magnetic field $\mathrm{B}_{\mathrm{ex}}$. Colors of $\mathrm{B}_{\mathrm{ex}}$ correspond to the measurement conditions of $\mathrm{d} \Omega_{\mathrm{SOI}} / \mathrm{d} r$ in (a). 


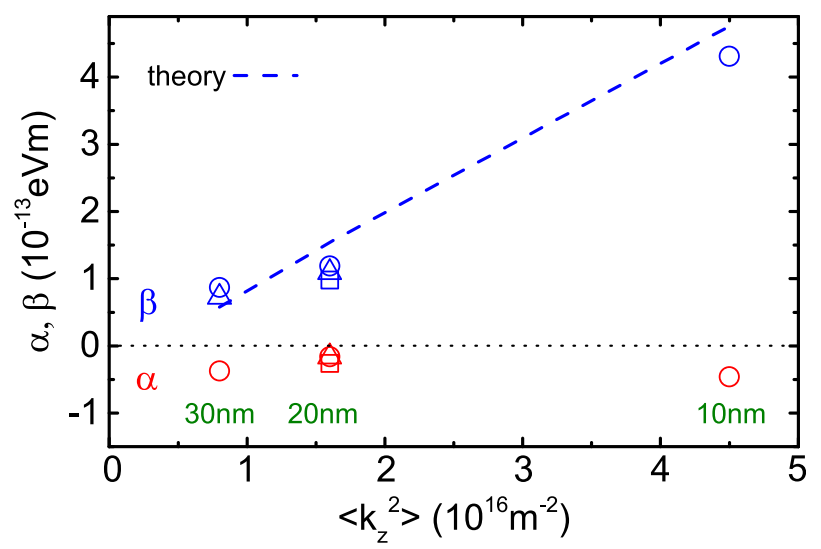

FIG. 5. Rashba and Dresselhaus SO coefficients, $\alpha$ and $\beta$, in $10-, 20-$, and 30-nm-wide GaAs QWs as a function of $\left\langle k_{z}^{2}\right\rangle$. Corresponding QW widths are indicated. Symbols show the results from the different measurement conditions: $B_{\mathrm{ex}}=+1 \mathrm{~T}$ along $x$ (circles), along $y$ (triangles), and along $-x$ (squares). The dashed line is a theoretical calculation of $\beta$ using the value of the bulk Dresselhaus coefficient $\gamma=-11 \mathrm{eV}^{3}$.

symbols), $x$-scans measure $B_{\mathrm{D}}$ and $y$-scans $B_{\mathrm{R}}$, but with different signs for the two directions of $B_{\mathrm{ex}}$. This is consistent with the measured slopes in Fig. 4(a) where we observe larger $\left(B_{\mathrm{D}}\right)$ and smaller $\left(B_{\mathrm{R}}\right)$ slopes. For $\mathbf{B}_{\mathrm{ex}} \| y$ (green symbols), $x$-scans measure $-B_{\mathrm{R}}$, and $y$-scans $+B_{\mathrm{D}}$. The $\beta$ and $\alpha$ evaluated are $1.09 \times 10^{-13}$ and $-1.6 \times 10^{-14} \mathrm{eVm}$ for $\mathbf{B}_{\mathrm{ex}} \|+y$, and $0.98 \times 10^{-13}$ and $-2.6 \times 10^{-14} \mathrm{eVm}$ for $\mathbf{B}_{\mathrm{ex}} \|-x$, respectively, and consistent with the results of $\mathbf{B}_{\mathrm{ex}} \|+x$. By considering the symmetry relation of $\mathbf{B}_{\mathrm{R}}$ and $\mathbf{B}_{\mathrm{D}}$ with $\mathbf{B}_{\mathrm{ex}}$, we unambiguously detect Rashba and Dresselhaus SO interactions.

We obtained the SO parameters in all QWs of width 10, 20 , and $30 \mathrm{~nm}$. We plot $\beta$ and $\alpha$ of the three samples as a function of $\left\langle k_{z}^{2}\right\rangle$ in Fig. 5. $\left\langle k_{z}^{2}\right\rangle$ was calculated taking wavefunction leakage into the barrier region into account. Circles, triangles, and squares correspond to the results from different symmetry measurements, i.e., $\mathbf{B}_{\mathrm{ex}}\left\|+x, \quad \mathbf{B}_{\mathrm{ex}}\right\|+y$, and $\mathbf{B}_{\mathrm{ex}} \|-x$, respectively. The evaluated $\beta$ increases with $\left\langle k_{z}^{2}\right\rangle$ and is well explained by $\beta=-\gamma\left\langle k_{z}^{2}\right\rangle+\frac{1}{4} \gamma k_{\mathrm{F}}^{2}$ with $\gamma=-11 \mathrm{eV \AA}^{3}$ (dashed line in Fig. 5), which is consistent with the result evaluated from drift-induced $\mathbf{B}_{\mathrm{D}}{ }^{18}$ and other experimental works. ${ }^{20,21}$ The $|\alpha|$ obtained is smaller than $\beta$ and does not depend systematically on $\left\langle k_{z}^{2}\right\rangle$. This is explained by the doping profile chosen, which leads to a small potential gradient across the GaAs/AlGaAs QW. ${ }^{17}$

In conclusion, we investigated the influence of Rashba and Dresselhaus SO-induced effective magnetic fields on the spin dynamics measured by spatially separated spinexcitation and spin-detection locations. By changing the relative position between the pump and probe spots as well as the pump spot size in a TRKR measurement, a spatial modulation of the spin precession frequency is observed that depends on the scan direction. This reflects the anisotropic SO field due to Rashba and Dresselhaus SO interactions and makes it possible to evaluate both SO coefficients without having to process sample structures or apply electrical currents.

We acknowledge financial support from the Swiss National Science Foundation through the National Center of Competence in Research (NCCR) QSIT, and from the Japanese Ministry of Education, Culture, Sports, Science, and Technology (MEXT) in Grant-in-Aid for Scientific Research Nos. 15H02099 and 25220604, as well as from the DFG (SFB 689). We thank R. Allenspach, A. Fuhrer, and T. Henn for useful discussions.

${ }^{1}$ D. D. Awschalom and M. E. Flatté, Nat. Phys. 3, 153 (2007).

${ }^{2}$ T. Jungwirth, J. Wunderlich, and K. Olejník, Nat. Mater. 11, 382 (2012).

${ }^{3}$ Y. A. Bychkov and E. I. Rashba, J. Phys. C: Solid State Phys. 17, 6039 (1984).

${ }^{4}$ G. Dresselhaus, Phys. Rev. 100, 580 (1955).

${ }^{5}$ Y. Kato, R. C. Myers, A. C. Gossard, and D. D. Awschalom, Nature 427, 50 (2004).

${ }^{6}$ S. A. Crooker and D. L. Smith, Phys. Rev. Lett. 94, 236601 (2005).

${ }^{7}$ L. Meier, G. Salis, I. Shorubalko, E. Gini, S. Schön, and K. Ensslin, Nat. Phys. 3, 650 (2007).

${ }^{8}$ M. Studer, G. Salis, K. Ensslin, D. C. Driscoll, and A. C. Gossard, Phys. Rev. Lett. 103, 027201 (2009).

${ }^{9}$ M. I. D'yakonov and V. I. Perel', Fiz. Tverd. Tela 13, 3581 (1971) [Sov. Phys. Solid State 13, 3023 (1972)].

${ }^{10}$ M. I. D'yakonov and V. Yu. Kachorovskii, Fiz. Tekh. Poluprovodn. 20, 178 (1986); [Sov. Phys. Semicond. 20, 110 (1986)].

${ }^{11}$ J. D. Koralek, C. P. Weber, J. Orenstein, B. A. Bernevig, Shou-Cheng Zhang, S. Mack, and D. D. Awschalom, Nature 458, 610 (2009).

${ }^{12}$ M. P. Walser, C. Reichl, W. Wegscheider, and G. Salis, Nat. Phys. 8, 757 (2012).

${ }^{13}$ J. Ishihara, M. Ono, Y. Ohno, and H. Ohno, Appl. Phys. Lett. 102, 212402 (2013).

${ }^{14}$ P. Altmann, M. P. Walser, C. Reichl, W. Wegscheider, and G. Salis, Phys. Rev. B 90, 201306(R) (2014).

${ }^{15}$ J. Ishihara, Y. Ohno, and H. Ohno, Jpn. J. Appl. Phys., Part 1 53, 04 EM04 (2014).

${ }^{16}$ S. D. Ganichev and L. E. Golub, Phys. Status Solidi B 251, 1801 (2014).

${ }^{17}$ V. Lechner, L. E. Golub, F. Lomakina, V. V. Bel'kov, P. Olbrich, S. Stachel, I. Caspers, M. Griesbeck, M. Kugler, M. J. Hirmer, T. Korn, C. Schüller, D. Schuh, W. Wegscheider, and S. D. Ganichev, Phys. Rev. B 83, 155313 (2011).

${ }^{18}$ M. P. Walser, U. Siegenthaler, V. Lechner, D. Schuh, S. D. Ganichev, W. Wegscheider, and G. Salis, Phys. Rev. B 86, 195309 (2012).

${ }^{19}$ Y. S. Chen, S. Fält, W. Wegscheider, and G. Salis, Phys. Rev. B 90, 121304(R) (2014).

${ }^{20}$ W. J. H. Leyland, R. T. Harley, M. Henini, A. J. Shields, I. Farrer, and D. A. Ritchie, Phys. Rev. B 76, 195305 (2007).

${ }^{21}$ P. S. Eldridge, J. Hübner, S. Oertel, R. T. Harley, M. Henini, and M. Oestreich, Phys. Rev. B 83, 041301 (2011). 